\title{
Erratum
}

\section{tert-Butoxide-Assisted Amidation of Esters under Green Conditions}

Bo Ram Kim, Hyung-Geun Lee, Seung-Beom Kang, Gi Hyeon Sung, Jeum-Jong Kim, Jong Keun Park,* Sang-Gyeong Lee, Yong-Jin Yoon* Synthesis 2012, 42.

One tert-butoxide radical in Scheme 2 of the advance online publication (e-First) version of this article contained an additional $\mathrm{H}$ atom, and the ${ }^{1} \mathrm{H}$ NMR data of compound $\mathbf{5 c}$ had a minor formatting error.

These mistakes have been corrected for both the current online version and the print publication. 